\title{
Differential sensitivity of skeletal and fusimotor neurons to Bcl-2-mediated apoptosis during neuromuscular development
}

\author{
K Hui $^{1}$, J Kucera ${ }^{2}$ and JT Henderson ${ }^{\star, 1}$
}

Proper development of the nervous system requires that a carefully controlled balance be maintained between both proliferation and neuronal survival. The process of programmed cell death is believed to play a key role in regulating levels of neuronal survival, in large part through the action of antiapoptotic proteins, such as $\mathrm{Bcl}-2$. Consistent with this, $\mathrm{Bcl}-2$ has been shown to be a key regulator of apoptotic signaling in post-mitotic neurons. However, we still know remarkably little regarding the role that $\mathrm{Bcl}-2$ plays in regulating the survival of specific motor neuron populations. In the present study, we have examined somatic motor neurons of the lumbar spinal cord, and branchiomotor neurons of the facial nucleus in $b c l-2$-null mice to determine the differential dependence among motor neuron populations with respect to $\mathrm{Bcl}-2$-mediated survival. Examination of neuronal and axon number, axonal area, and the distribution of axonal loss in bcl-2-null mice demonstrates that, in contrast to the great majority of alpha motor neurons, gamma motor neurons exhibit a unique dependence upon bcl-2 for survival. These results demonstrate, for the first time, the connection between Bcl-2 expression, motor neuron survival, and the establishment of different motor populations.

Cell Death and Differentiation (2008) 15, 691-699; doi:10.1038/sj.cdd.4402294; published online 21 December 2007

Within the central nervous system (CNS), more post-mitotic neurons are produced than are ultimately incorporated into the mature synaptic architecture. ${ }^{1}$ In the motor system, this is believed to, in part, reflect competition among developing neurons for limited levels of neurotrophic support. ${ }^{1,2}$ Neurons failing to achieve optimal innervation are in turn eliminated during the latter embryonic and early postnatal periods. ${ }^{1}$ Numerous studies have demonstrated the critical role which programmed cell death (PCD) plays in this process. ${ }^{3}$ While many stimuli have been shown to be capable of initiating PCD, the core components of this process have been shown to be highly conserved among species as divergent as Caenorhabditis elegans and human. In mammals, two core PCD pathways, termed the extrinsic and intrinsic pathways, have been identified. ${ }^{4} \mathrm{~A}$ major point of convergence between these pathways exists at the mitochondria. It is here, that interaction between pro- and antiapoptotic $\mathrm{Bcl}-2$ family members determines subsequent PCD progression through permeabilization of the mitochondrial outer membrane, inducing both caspase-dependent and caspase-independent signaling. ${ }^{5,6} \mathrm{Bcl}-2$ is the prototypical antiapoptotic $\mathrm{Bcl}-2$ family member, a family that includes Bcl- $\mathrm{x}_{\mathrm{L}}, \mathrm{Bcl}-\mathrm{w}, \mathrm{Mcl}-1, \mathrm{~A} 1$, Blf-1, boo/diva, Nr-13, and Ced-9. ${ }^{7}$ While questions remain regarding the precise mechanism by which they inhibit PCD, evidence suggests that interference with the oligomerization and/or conformational activation of proapoptotic proteins, such as Bax and Bak is critical. ${ }^{8} \mathrm{Bcl}-2$ is also known to interact with components of the mitochondrial permeability transition pore, which is minimally comprised of voltage-dependent anion channel, adenine nucleotide translocase, and cyclophilin $D$ to alter mitochondrial membrane potential. ${ }^{9}$ Because of these varied functions, and results from previous knockout and overexpression studies in vivo, $\mathrm{Bcl}-2$ has been identified as a key PCD regulator within both developing and postnatal neurons in mammals. ${ }^{3}$

During development, $\mathrm{Bcl}-2$ and $\mathrm{Bcl}-\mathrm{x}_{\mathrm{L}}$ in particular, are widely expressed within the CNS both during and following neurogenesis. ${ }^{10-12}$ Consistent with this, both proteins are thought to play a role in regulating naturally occurring cell death (NOCD). The expression pattern of these antiapoptotic proteins differs, however, with respect to latter development. $\mathrm{Bcl}-\mathrm{x}_{\mathrm{L}}$ continues to be widely expressed in many regions of the postnatal CNS, whereas bcl-2 expression declines in many CNS regions postnatally. ${ }^{10,12-14}$ However, some neuronal populations do maintain $b c /-2$ expression postnatally. These include neurons of the sympathetic and sensory ganglia, ${ }^{12,15,16}$ granule cells of the cerebellum and dentate gyrus, ${ }^{10}$ branchiomotor neurons, ${ }^{15}$ and motor and interneurons of the spinal cord. ${ }^{12,14,15,17}$ With respect to motor neurons, a previous study has suggested that differential sensitivity to $\mathrm{bcl}-2$ deletion may exist within branchiomotor neurons of the facial nucleus. ${ }^{15}$

Several independent lines of $b c l-2$-null mice have been generated previously, eliminating either the $b c l-2 \alpha^{18}$ or

${ }^{1}$ Graduate Department of Pharmaceutical Sciences, Leslie Dan Faculty of Pharmacy, University of Toronto, Ontario, Canada and ${ }^{2}$ Neurology Research, VA Medical Center, Boston, MA, USA

${ }^{*}$ Corresponding author: JT Henderson, Graduate Department of Pharmceutical Sciences, Leslie Dan Faculty of Pharmacy, University of Toronto, 144 College Street, Room 903, Toronto, Ontario, Canada M5S 3M2. Tel: 416946 3090; Fax: 416978 8511; E-mail: jeff.henderson@ utoronto.ca

Keywords: Bcl-2; motor neuron; development; apoptosis

Abbreviations: ChAT, choline acetyltransferase; CNS, central nervous system; DRG, dorsal root ganglion; GDNF, glial cell line-derived neurotrophic factor; MFN, medial facial nerve; NOCD, naturally occurring cell death; NT-3, neurotrophin-3; PCD, programmed cell death

Received 13.7.07; revised 25.10.07; accepted 09.11.07; Edited by N Bazan; published online 21.12.07 
$b c l-2 \alpha / \beta^{15,19}$ transcripts. Each of these lines shows essentially identical phenotypes. Bcl-2-null mice have been shown to exhibit a reduced growth rate, reduced lifespan (largely due to polycystic kidneys), reduced T-cell lifespan, craniofacial anomalies, and hair graying during the second follicle cycle. $^{15,18,19}$ With respect to the CNS, no gross developmental anomalies have been described. Bcl-2-null mice have been shown to exhibit some developmental loss of sensory, sympathetic, and branchiomotor neurons during the early postnatal period. ${ }^{15}$ Similarly, some postnatal loss of retinal ganglion cells has been observed in bcl-2-null mice between $\mathrm{P} 10$ and $\mathrm{P} 15$, beyond the period of NOCD. ${ }^{20}$ In vitro analyses of sympathetic and trigeminal neurons have suggested a role for Bcl-2 in NOCD. ${ }^{16,21}$ With respect to effects on injuryrelated survival, in vivo transection of both the optic ${ }^{22}$ and facial $^{15}$ nerves shows no difference in survival in $b c l-2-n u l l$ mice versus controls. Similarly, bcl-2-null mice exhibit no difference compared to controls in either neurotrophinmediated survival in vitro ${ }^{21}$ or following acute injury. ${ }^{15}$

In order to determine the effects of Bcl-2 deficiency on various motor neuron populations in vivo, we have examined the properties of lumbar motor neurons of the spinal cord and branchiomotor neurons of the facial nucleus in several strains of $b c l-2-n u l l$ mice. The results demonstrate for the first time that $\mathrm{Bcl}-2$ plays a unique and critical role in the survival of gamma motor neurons generated during the latter part of neurogenesis.

\section{Results}

Bcl-2 differentially regulates the survival of spinal motor neurons. To examine the effect of $\mathrm{Bcl}-2$ deficiency on lumbar spinal motor neurons, total counts of motor neurons were performed on choline acetyltransferase (ChAT) and Nissl-stained serial sections. To first determine those spinal levels which contribute motor axons to the sciatic nerve in bcl-2-null mice and controls, retrograde tracing of sciatic nerves was performed for each genotype. As shown in Figure $1 \mathrm{a}$ and $\mathrm{b}, \mathrm{bcl}-2-$ null mice exhibit a comparable distribution of lumbar spinal motor neurons with respect to both rostral-caudal and intralaminar distribution, respectively, compared to control littermates. As shown in Figure 1c, comparisons of ChAT-positive motor neurons from sciatic levels T13-L2 demonstrate that bcl-2-null mice exhibit a significant reduction $(26 \pm 3 \%)$ in total motor neuron number at postnatal day 30 (P30) compared to control littermates. Counts of spinal motor neuron number performed on additional (alternate) Nissl-stained sections demonstrated comparable levels of motor neuron loss, indicating that bcl-2 ablation does not affect ChAT expression. These results demonstrate that $b c /-2$ deficiency results in the loss of a sub-population of sciatic motor neurons.

To examine the pattern of $\mathrm{Bcl}-2$ expression within spinal motor neurons, spinal cord sections through the sciatic motor pool were stained for $\mathrm{Bcl}-2$ in the early postnatal period. As shown in Figure $2 \mathrm{a}-\mathrm{c}$, a minor population of motor neurons exhibited persistent expression of Bcl-2 postnatally. Stereotactic analysis of wild-type mice within sciatic spinal levels indicated that $32 \pm 8 \%$ of motor neurons within this region expressed $\mathrm{Bcl}-2$ postnatally.
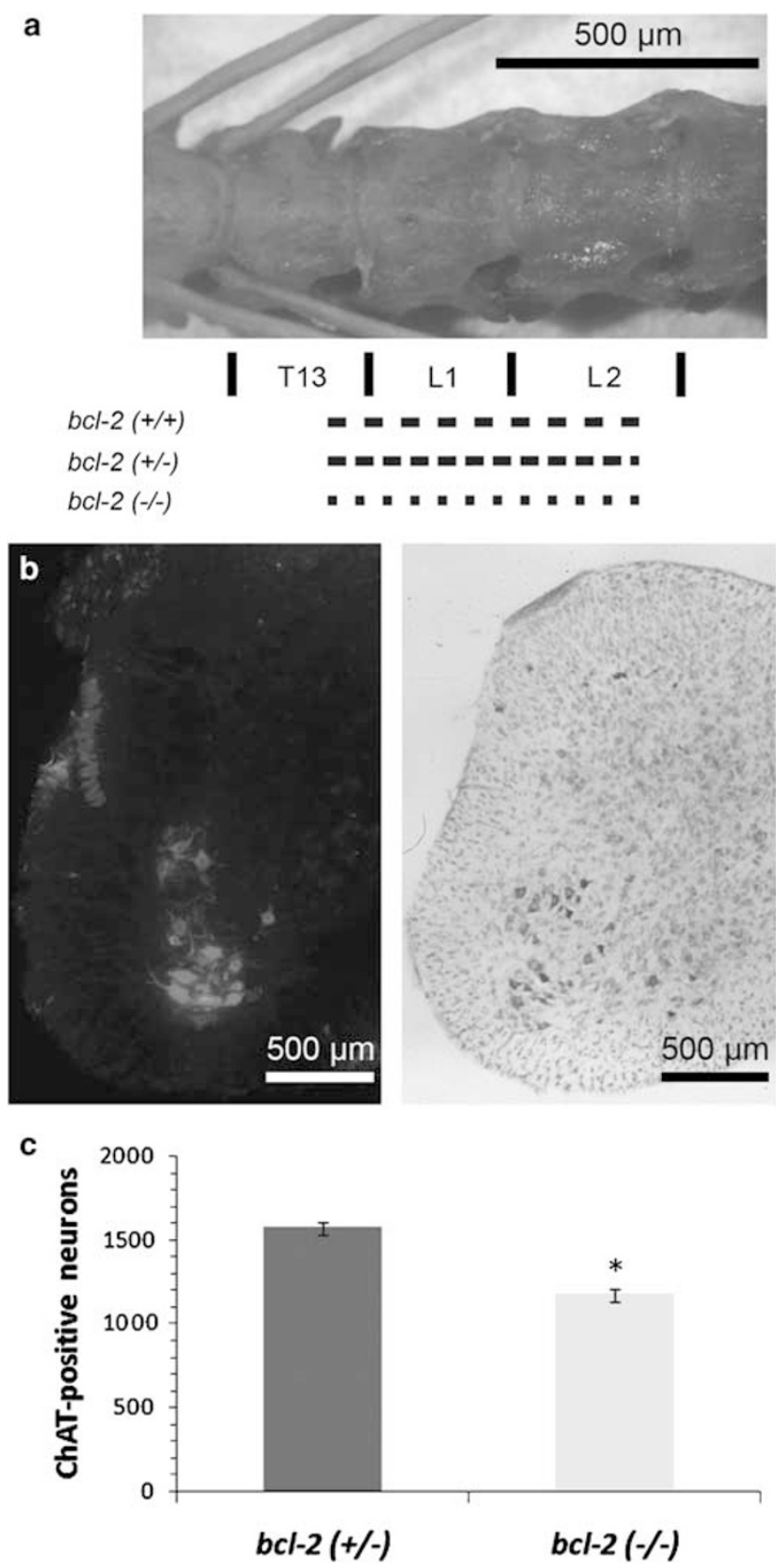

Figure 1 Properties of sciatic motor pool in bcl-2-null mice. The distribution of motor neurons comprising the sciatic pool was examined in bcl-2-null mice and controls. (a) Summary of rostral-caudal distribution of labeled motor neurons from sciatic nerve with respect to spinal level in bcl-2 wild-type, heterozygous, and null mice ( $n=5$ animals per genotype). Spinal segments are indicated for reference. (b) Distribution of sciatic motor neurons in bcl-2-null mice. Left panel shows a typical pattern of motor neuron labeling observed $48 \mathrm{~h}$ following the application of rhodamine dextran to sciatic nerve stumps transected $3 \mathrm{~mm}$ distal to the obdurator tendon in $b c l-2^{-1-}$ mice (spinal level $=\mathrm{L} 1$ ). Right panel shows a thionin-stained section at similar level highlighting Nissl-stained neurons of the sciatic motor pool. (c) Histogram of ChAT-positive neurons with spinal levels contributing to sciatic motor pools (T13-L2) in bcl-2 ${ }^{+/-}$and $b c l-2^{-1-}$ mice

To better understand the nature of the motor neuron loss seen in $b c /-2-$ null mice, $1 \mu \mathrm{m}$ thin sections were cut through the $L 4$ ventral roots of $b c l-2^{-/-}, b c l-2^{+/-}$, and $b c l-2^{+/+}$mice at $P 30$. As shown in Figure $3 a$ and b, within the $L 4$ ventral root, 

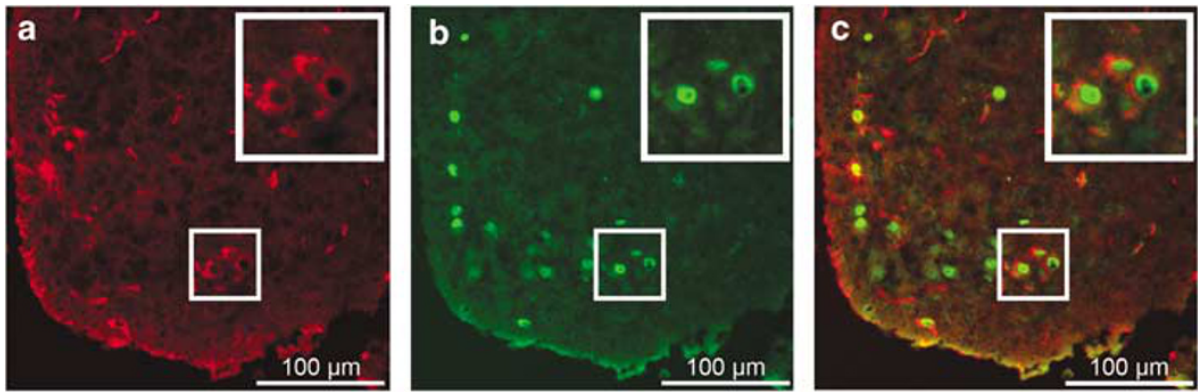

Figure 2 Persistent expression of Bcl-2 in a sub-population of spinal motor neurons. The pattern of immunohistochemical staining for Bcl-2 (red) and Islet-1/2 (green) was examined in wild-type mice at P1. Islet-1/2 staining was used as an independent marker to delineate motor neurons within lamina IX and X at P1. (a) Pattern of Bcl-2 staining, (b) Islet-1/2 staining, (c) Bcl-2, and Islet-1/2 overlay. Insets show enlargement of region indicated, showing double-labeled motor neurons expressing Bcl-2. Scale bar represents a distance of $50 \mu \mathrm{m}$. Note that only a sub-population of lumbar motor neurons expresses Bcl-2 at this stage

a

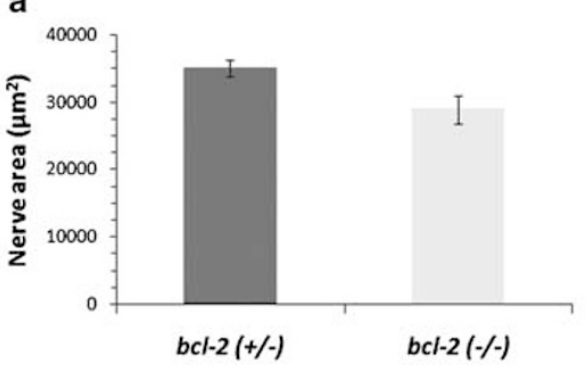

C

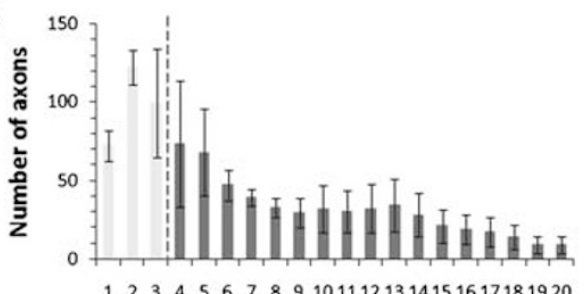

Axon area $\left(\mu \mathrm{m}^{2}\right)$

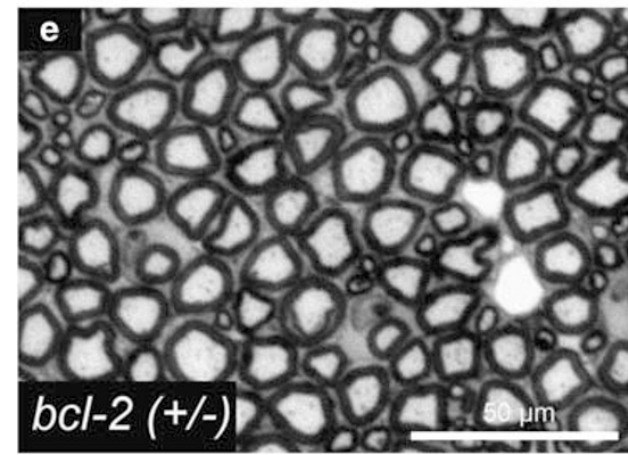

b

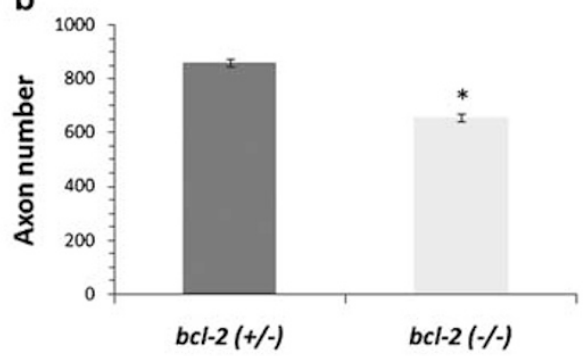

d

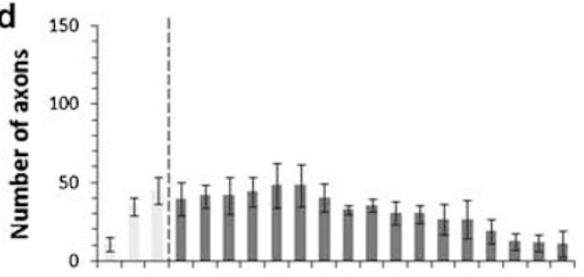

12345567891011121314151617181920 Axon area $\left(\mu \mathrm{m}^{2}\right)$

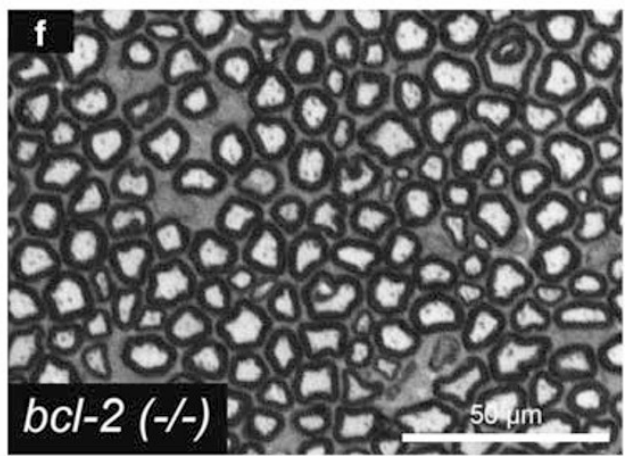

Figure 3 Analysis of motor axon populations in bcl-2-null mice. Shown are the properties of motor axons in the L4 ventral root in bcl-2-null mice and controls. (a) Total cross-sectional area of the $L 4$ ventral roots in $b c l-2$-null mice and controls. Areas are indicated in $\mu \mathrm{m}^{2} . N=7$ and 6 for $b c l-2(+/-)$ and $(-/-)$ groups, respectively. Note that with respect to total L4 ventral root area, groups did not significantly different from one another $(P=0.14)$. (b) Total axon numbers within the $L 4$ ventral root. $N=7$ and 6 for $(+I-)$ and $(-I-)$ groups, respectively. S.E.M. equal to 15 and 16 for $(+I-)$ and $(-I-)$ groups, respectively. Asterisk denotes significant difference from control group at $P<0.01$. (c) Histogram showing the distribution of axon areas within L4 ventral roots of $b c l-2^{+l-}$ mice. Histogram represents the axon areas observed in complete L4 crosssections ( $n$ animal $\geqslant 5, n$ axon $\geqslant 4300$ ). (d) Histogram distribution for $b c l-2$-null mice ( $n$ animal $\geqslant 5, n$ axons $\geqslant 3300)$. For both (c) and (d) approximately $\geqslant 95 \%$ of all axon areas fall within the range indicated on the graph, with $<5 \%$ exhibiting areas greater than the indicated range. (e-f) Photomicrographs of $L 4$ ventral root of $b c l-22^{+/-}$and $b c l-2^{-1-}$ L4 ventral roots, respectively at P30 demonstrating loss of small caliber axons. Scale bar represents a distance of $50 \mu \mathrm{m}$

total axon counts demonstrated that $b c /$-2-null mice exhibited a small but significant reduction in axon number compared to littermate controls, despite no significant reduction in total nerve area. Interestingly, the percent reduction in axon number (24 $\pm 2 \%$ ) seen in bcl-2-null mice was similar to that observed for spinal motor neurons expressing Bcl-2 postnatally. 
To examine this relationship in greater detail, the distribution of axon cross-sectional areas was determined in entire cross-sections of L4 ventral roots. Individual axon crosssectional areas were determined rather than the standard practice of measuring axon diameter, due to its inherently greater reliability. As shown in Figure $3 c$ and d, bcl-2-null mice exhibit a preferential loss of small caliber axons $\left(\leqslant 3 \mu \mathrm{m}^{2}\right)$. The percent loss of small caliber axons was $61 \pm 2 \%$ compared to a $12 \pm 3 \%$ reduction in larger $\left(>3 \mu \mathrm{m}^{2}\right)$ caliber axons in bcl-2-null mice. Analysis of L4 ventral roots, as shown in Figure $3 \mathrm{e}$ and $\mathrm{f}$, demonstrated that $\mathrm{bcl}-2^{-1-}$ mice exhibit a dramatic reduction in small caliber axons compared to heterozygous $b c /-2$ littermates. The extent of this axonal reduction $(24 \pm 2 \%)$ strongly correlated with the magnitude of the reduction in ChAT-positive neurons $(26 \pm 3 \%)$ seen in sciatic spinal levels, and the population of motor neurons expressing Bcl-2 postnatally (32 $\pm 8 \%$ ). These data indicate that the loss of motor axons seen in bcl-2-null mice occurs as a consequence of motor neuron death.

Previous studies have suggested that the neuronal loss resulting from the ablation of $b c l-2$ occurs during the late embryonic/early postnatal periods, with neuronal reductions stabilizing around P9. ${ }^{15}$ To determine whether this pattern was also observed in our lineages of $b c /-2$ mice, bcl-2-null mice and controls were examined at P10. Counts of $L 4$ ventral roots at $\mathrm{P} 10$ indicated an $18 \pm 2 \%$ reduction in $\mathrm{bcl}-2-$-null mice compared to controls (axon means: $694 \pm 11$ versus $848 \pm 9$ for controls).

Reduction of muscle spindle innervation and sensory losses in bcl-2-null mice. The loss of small caliber motor axons seen in bcl-2-null mice indicated that gamma motor neurons are uniquely sensitive to developmental ablation of $\mathrm{Bcl}-2$. As a result, we examined the fusimotor innervation of muscle spindles. As this structure also receives sensory innervation, sensory axons were also examined in bcl-2-null mice. As shown in Figure $4 a$ and $b$, both nerve area and axon number were reduced within the dorsal root ganglion (DRG) by $28 \pm 9$ and $27 \pm 2 \%$, respectively, suggesting that $\mathrm{bcl}-2^{-/-}$ mice undergo a significant loss of sensory neurons within the DRG by P30. Consistent with this, prior analysis of the L3 $\mathrm{DRG}$ in the $b c /-2^{-1}$ mice has demonstrated significant neuronal loss at P9. ${ }^{15}$

To more clearly define the effects of sensory and motor axon reduction on muscle spindles, the number and morphology of muscle spindles were examined in bcl-2-null mice. Total numbers of muscle spindles were determined in serial cross-sections of both forelimb (flexor carpi ulnaris) and hindlimb (soleus) muscles. At both sites, spindle number did not differ significantly between genotypes. Similarly, the cross-sectional area of intrafusal $\left(\right.$ bag $_{2}$ ) fibers, or adjacent extrafusal (skeletal) muscle fibers of $b c l-2^{-1-}$ and $b c l-2^{+/-}$ mice did not differ significantly (intrafusal: $b c l-2^{-1-} 28 \pm 3 \mu \mathrm{m}^{2}$, $b c l-2^{+l-} 22 \pm 4 \mu \mathrm{m}^{2}, n=40$ fibers per genotype; extrafusal: $b c l-2^{-l-} 219 \pm 17 \mu \mathrm{m}^{2}, b c l-2^{+l-} 225 \pm 22 \mu \mathrm{m}^{2}, n=80$ fibers per genotype). To determine the level of fusimotor innervation of muscle spindles in $b c /-2^{-/-}$mice, soleus muscle spindles were cut through both the central (capsular) and terminal regions. As shown in Figure 4c, soleus muscle spindles from $b c l-2$ heterozygous and wild-type mice receive innervation from multiple fusimotor axons, while spindles from $b c /-2^{-1-}$ mice are typically innervated by only a single apparent motor
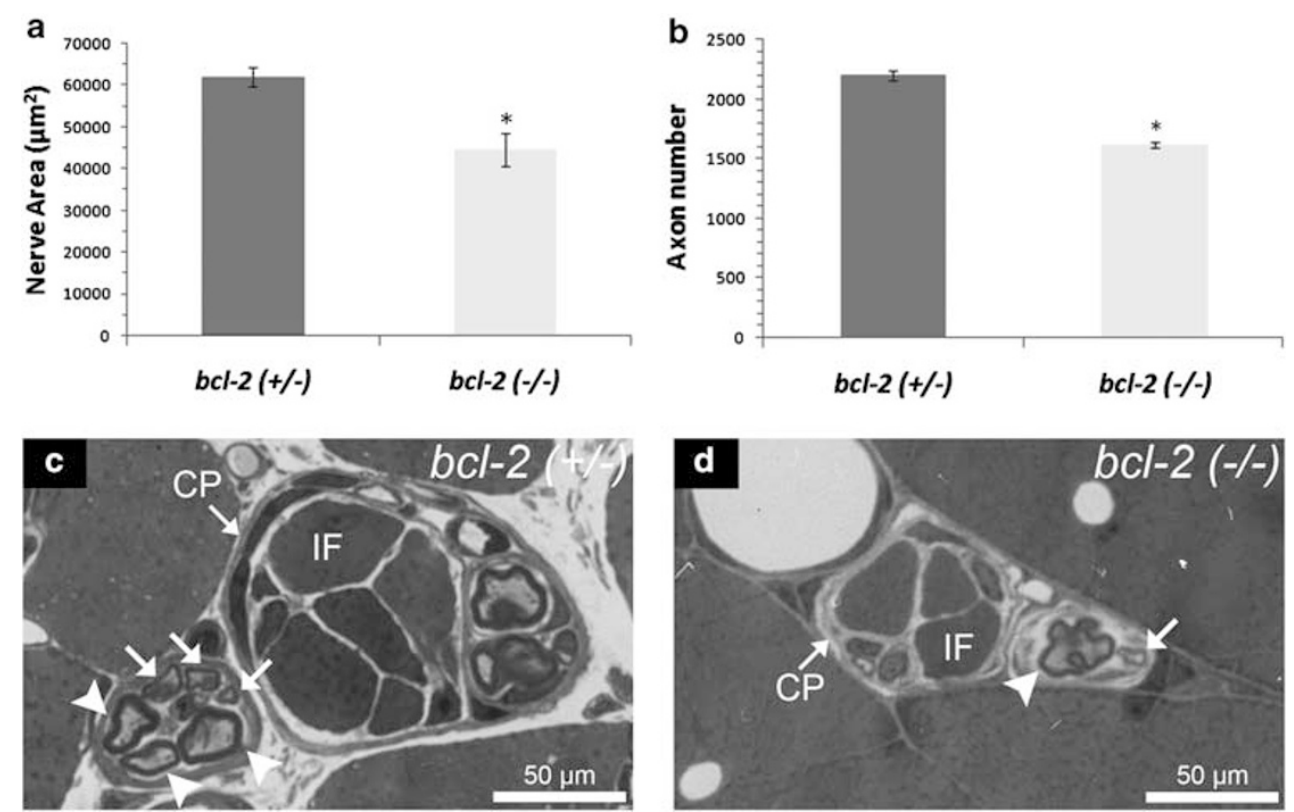

Figure 4 Loss of $b c l-2$ alters sensory and muscle spindles innervation. The extent of reduction in sensory and muscle spindle innervation was determined for $b c l-2-n u l l$ mice. (a) Cross-sectional area of L4 dorsal roots in $b c l-2^{+-}$and $b c l-2^{-1-}$ mice at P3O. (b) Total axon counts of L4 dorsal root of $b c l-2^{+l-}$ and $b c l-2^{-1-}$ mice at P30 $(n \geqslant 5$ for each genotype; asterisk indicates significant difference from the control group at $P<0.01)$. (c) Example of soleus muscle spindle in bcl-2 ${ }^{+l-}$ mouse. Note that the spindle receives a large la sensory axon, two smaller secondary sensory axons (arrowheads), and three small fusimotor axons (arrows). Intrafusal fibers within the spindle are denoted by IF, which are surrounded by spindle capsule (CP). Extrafusal skeletal muscle fibers surround the spindle capsule and neural axons. (d) Example of soleus muscle spindles in $b c l-2^{-l-}$ mouse. Note that $b c l-2^{-l-}$ spindle receives an la axon (arrowhead), and a single apparent fusimotor axon (arrow) with no secondary sensory axons. For (c) and (d), scale bar denotes $50 \mu \mathrm{m}$ 
axon (Figure 4d). Counts of total fusimotor fibers within the soleus muscle indicate a significant decrease in fusimotor innervation in $b c /-2^{-1-}$ mice versus controls $(15 \pm 4$ versus $80 \pm 6$ ). Thus, while $b c l-2-n u l l$ mice exhibit a substantial loss of fusimotor innervation, some residual motor innervation to muscle spindles remained in these animals. Consistent with this, esterase activity is observed within at least some muscle spindles in bcl-2-null mice (data not shown). This innervation may represent the influence of either a small population of surviving fusimotor fibers or collateral sprouting from existing alpha motor fibers (beta innervation). This residual motor innervation may explain the absence of gross dystonia or ataxia seen in bcl-2-null mice. Examination of soleus muscle termini in bcl-2-null mice also demonstrated the presence of normal golgi tendon organs.

Role of $\mathbf{b c l}-2$ in PCD in the facial nucleus. To compare the results observed in spinal motor neurons, branchiomotor neurons of the facial nucleus were examined in bcl-2-null mice. This population was chosen for analyses both because it lacks significant gamma motor innervation ${ }^{23}$ and because it has been examined previously in lines of $b c /-2-n u l l$ mice. ${ }^{15}$ In addition, we wished to examine motor neuron survival within lateral components of the facial nucleus, as these have previously been suggested to undergo preferential degeneration in bcl-2-null mice. To analyze the lateral (nasolabial) component of the facial nucleus, a retrograde tracer was injected into the medial facial nerve (MFN) (Figure $5 \mathrm{a}$ ) to examine this sub-population within the facial nucleus proper (overview, Figure $5 b$ ) for each genotype. As shown in Figure $5 c$ and $d$, motor neurons, which innervate the MFN, reside within the lateral portion of the facial nucleus. Retrograde labeling of the MFN in bcl-2-null mice demonstrated no significant difference in motor neuron distribution compared to control littermates. It should be noted, however, that a small group of motor neurons at the extreme lateral extent of the facial nucleus was not labeled following MFN injection in any of the genotypes examined.

To determine the overall impact of $b c /-2$ deletion on facial motor neurons, total counts of motor neurons within the facial nucleus were performed in P30 bcl-2-null mice and controls. As shown in Figure $5 \mathrm{e}$, our lineages of bcl-2-null mice exhibited a reduction of $21 \pm 2 \%$ in facial motor neurons compared to controls. Interestingly, it has previously been suggested that $b c /-2-n u l l$ mice exhibit an enhanced degeneration of motor neurons within the lateral component of the facial nucleus. ${ }^{15}$ To investigate this in our lines of $b c l-2-$-null mice, cross-sectional area, total axon number, and distribution of axon calibers within the MFN were determined for bcl-2-null mice and controls. As shown in Figure $6 a$ and b, bcl-2-null mice exhibited a reduction in both total axon number $(15 \pm 3 \%)$ and mean cross-sectional area (39 $\pm 6 \%)$ compared to controls at P30. In addition, the distribution of axon calibers was shifted toward smaller axon sizes in bcl-2-null mice compared to controls (Figure $6 \mathrm{c}$ and d). Axons within the MFN having cross-sectional areas $\leqslant 3 \mu \mathrm{m}^{2}$ represent $49 \pm 6 \%$ of the total axon population in $b c /-2-n u l l$ mice, whereas this group represents only $21 \pm 11 \%$ of the total axon population in $b c l-2$ heterozygous or wild-type littermates. Given that the facial motor neurons exhibited a total reduction of $21 \pm 2 \%$ and
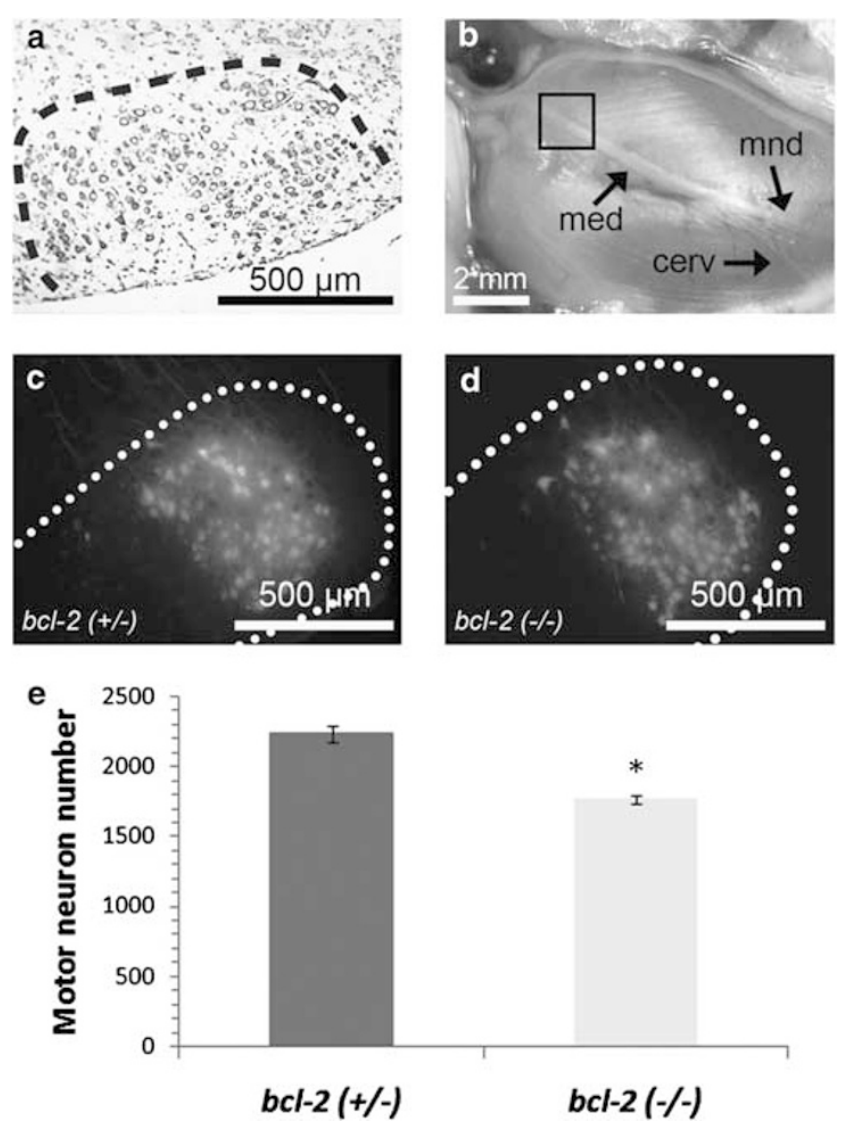

Figure 5 Effects of $b c l-2$ in facial motor neurons. Total and sub-populations of the facial nucleus analyzed in bcl-2-null mice and controls. (a) Overview of medial branches of the facial nerve showing retrograde-tracing site, and point of segment collection for the MFN (boxed region) used for analysis of axon morphometry (med, medial branch of the facial nerve, cerv, cervical branch of the facial nerve, mnd, mandibular branch of the facial nerve). (b) Photomicrograph showing coronal section of the brainstem delineating motor neurons within the facial nucleus (dotted zone). (c-d) Photomicrographs showing the pattern of retrograde labeling within the facial nucleus, following tracer injection to the MFN. Shown are typical distribution patterns for $b c l-2^{+1-}$ (c) and $b c l-2^{-1-}$ (d) mice $48 \mathrm{~h}$ following injection of tracer ( $n=5$ animals per genotype, labeled facial motor neurons $\geqslant 1220$ neurons per genotype, control labeling confined to lateral $35 \%$ of the facial nucleus). (e) Counts of total motor neuron number within the facial nucleus of $b c l-2^{+/-}$and $b c l-2^{-1-}$ mice. Counts were based upon $7 \mu \mathrm{m}$ serial sections taken through the full extent of the nucleus, with every sixth section analyzed for motor neuron number as described in Materials and Methods $(n=7$ and 6 for $(+/-)$ and $(-1-)$ groups, respectively). S.E.M. equal to 63 and 41 for $(+/-)$ and $(-/-)$ groups, respectively. Asterisk indicates significance at $P<0.01$

counts of retrogradely labeled (MFN) lateral facial neurons showed a reduction of only $17 \pm 3 \% \quad(1359 \pm 42$ versus $1643 \pm 73$ for controls, $n=5$ animals per genotype) in bcl-2null mice versus controls at P30, these data suggest that motor neurons with the lateral facial nucleus do not exhibit a differential sensitivity to $b c /-2$ ablation; in contrast to previous findings at P28. ${ }^{15}$

\section{Discussion}

To determine the effect of $b c l-2$ deletion on motor neuron survival, we have examined two motor populations that differ 

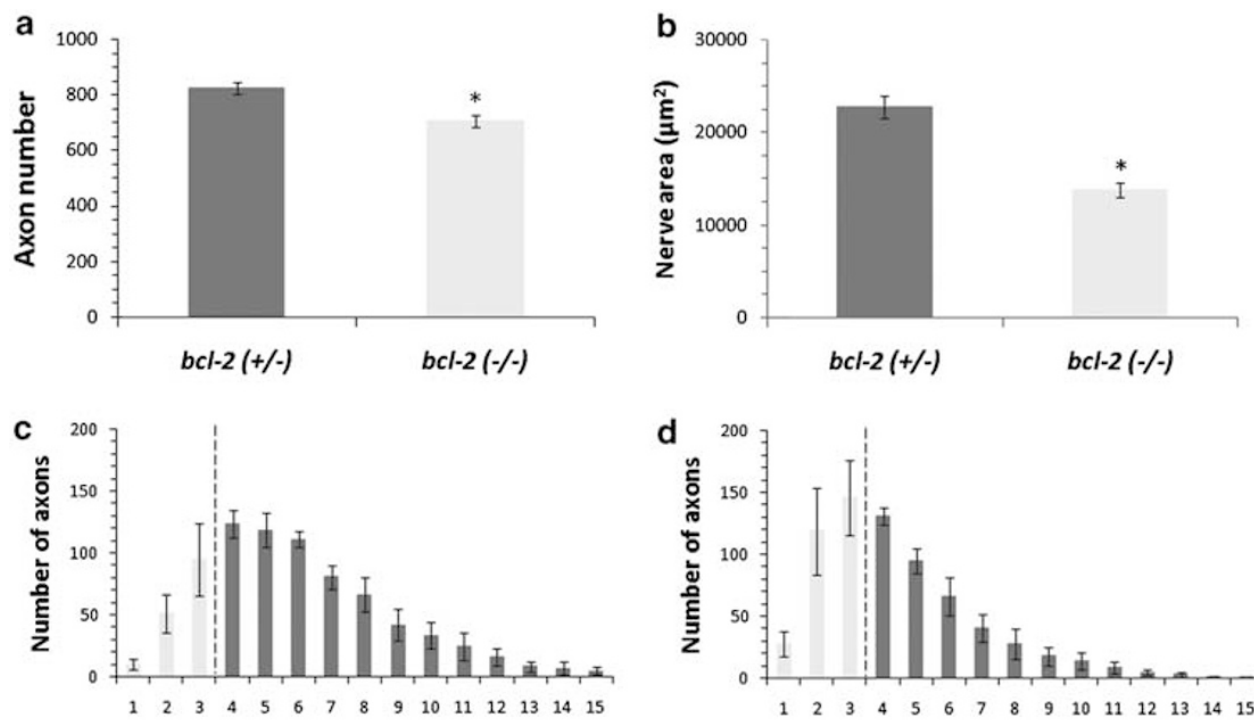

Axon area $\left(\mu \mathrm{m}^{2}\right)$

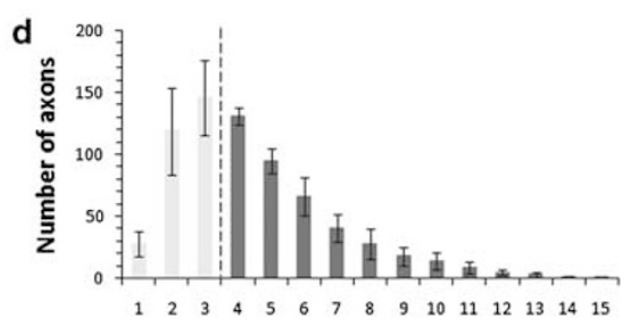

Axon area $\left(\mu \mathrm{m}^{2}\right)$

Figure 6 Analysis of axon/nerve morphology within the MFN in bcl-2-null mice. The morphology MFN was examined to assess the influence of bcl-2 upon this subpopulation of facial motor neurons. (a) Total axon numbers within the MFN $(n=8$ and 6 for $(+/-)$ and $(-/-)$ groups, respectively; S.E.M. equal to 23 and 22 for $(+/-)$ and $(-l-)$ groups, respectively). Asterisk indicates significance at $P<0.01$. (b) Total cross-sectional area of the MFN. Areas are indicated in $\mu \mathrm{m}^{2}, n=10$ and 8 for $(+/-)$ and $(-/-)$ groups, respectively. S.E.M. equal to 1201 and 814 for $(+/-)$ and $(-/-)$ groups, respectively. Asterisk indicates significant difference from control group at $P<0.01$. (c) Histogram showing distribution of axon caliber within the MFN in bcl-2 ${ }^{+/-}$mice. Histogram represents the axon areas observed in complete cross-sections of $b c l-2^{+/-}$ animals ( $n$ animal $\geqslant 5, n$ axon $\geqslant 4120$ ). (d) Distribution of axon caliber within MFN of $b c l-2$-null mice ( $n$ animal $\geqslant 5, n$ axons $\geqslant 3520)$. For histograms (c) and (d), $\geqslant 95 \%$ of all axon areas fall within the ranges indicated, with $<5 \%$ of axons exhibiting areas greater than the indicated range

significantly with respect to their fusimotor representation: branchiomotor neurons of the facial nucleus and somatic motor neurons of the lumbar spinal cord. Analyses of motor neurons in the lumbar spinal cord, and total axon numbers within the L4 ventral root indicated that $b c l-2^{-1-}$ mice exhibit significant motor neuron loss (24-26\%) compared to control littermates by P30. Retrograde tracings of the sciatic nerve to determine sciatic motor pool populations in $b c l-2^{-1-}, b c l-2^{+/-}$, and $b c l-2^{+1+}$ mice demonstrate equivalent motor representations between genotypes. Analysis of total axon crosssectional areas within L4 ventral roots demonstrate that the axonal losses observed in $b c /-2^{-1-}$ animals are not evenly distributed with respect to axon caliber, but disproportionately affect small caliber $\left(\leqslant 3 \mu \mathrm{m}^{2}\right)$ axons, indicating that small (gamma) motor neurons are selectively dependent on Bcl-2. Indeed, in terms of axon classes affected, $68 \%$ of the reduction observed in $b c /-2$-null mice affects axons of areas $\leqslant 3 \mu \mathrm{m}^{2}$. The predominant loss in small caliber axons seen in bcl-2-null mice corresponds to an extensive reduction in motor innervation (approximately $80 \%$ ) within muscle spindles as demonstrated from analysis of the soleus muscle. Residual motor input to muscle spindles may represent innervation from either a small number of surviving fusimotor fibers or induced collateral input from adjacent alpha motor neurons (beta fibers innervation).

In addition to spinal motor neurons, similar to a previous report, ${ }^{15}$ we observed losses of both branchiomotor and sensory neurons in bcl-2-null mice. However, the degree of neuronal loss we observe in our lineages of $b c /-2-n u l l$ mice on two different genetic backgrounds is somewhat lower (21 $\pm 2 \%$ P30 versus $32 \%$ P28 for facial motor neurons;
$27 \pm 2 \%$ P30 versus $44 \%$ P44 for sensory neurons). In addition, our analyses of the medial branch of the facial nerve (nasolabial musculature) suggest that this population of facial motor neurons is not differentially sensitive to $b c /-2$ ablation as reported previously. ${ }^{15}$ While the nature of this discrepancy remains unclear, it may be related to genetic differences in the murine backgrounds employed. Such differences are unlikely to be related to minor differences in the age of the animals employed (P30 versus P44), as both studies observed little increase in motor neuron due to $b c /-2$ deletion death beyond $\mathrm{Pg}$.

Within sciatic motor pools, previous analyses ${ }^{24}$ and our own studies place the fusimotor representation at approximately $30 \%$ of the total motor neuron population. Consistent with these findings, we observe that the population of lumbar spinal motor neurons that strongly express $\mathrm{Bcl}-2$ postnatally is comparable in number to both the degree of motor neuron loss seen in bcl-2-null mice at P30 and the fusimotor representation in this population. Alpha and gamma motor neurons represent two closely related lineages derived from a common neural precursor. ${ }^{25}$ Initially, both neural types make similar innervations to primary myofibers. ${ }^{26}$ A primary difference between these two motor classes relates to the temporal pattern of their differentiation. ${ }^{27}$ The majority of motor neurons differentiate during the early phase of spinal cord development (embryonic day 10-11 in rodents), and primary motor neurogenesis is essentially complete by E14. ${ }^{28}$ Alpha motor neurons (and interestingly group la sensory neurons, which provide innervation to muscle spindles) differentiate and become established early in this process. By contrast, differentiation of gamma motor neuron (and group II sensory 
neuron) occurs later, as the earliest known antigenic markers of these populations are observed beginning at E16.5-E17. ${ }^{27}$ Thus, it appears (at least within somatic motor pools) that the latter differentiation events of gamma motor neurons set the stage for enhanced dependence upon bcl-2 for survival, in contrast to earlier differentiation for alpha motor neurons. Thus, the differentiation of motor neurons into distinct classes occurs over the same developmental period that motor neuron numbers are principally adjusted through the process of PCD, from the peak levels of genesis to functional levels seen postnatally. Our results in bcl-2-null mice with spinal cord motor neurons demonstrate that these two processes can be mechanistically linked, guiding the development of specific motor populations in mammals in vivo.

It is interesting to note that ablation of $b c l-2$ not only influences gamma motor neurons but also results in the loss of a sub-population of alpha motor neurons in both lumbar spinal $\left(12 \pm 3 \%\right.$ based upon loss of axons $\left.>3 \mu \mathrm{m}^{2}\right)$ and branchiomotor $(21 \pm 2 \%$ based upon total facial nucleus counts) neurons. It is tempting to speculate that these bcl-2dependent alpha motor neurons may represent latterdifferentiating members of the population. The concept of a temporal pattern for $b c l-2$ in regulating neuronal survival is consistent with our observation that neither muscle spindle number (regulated by la sensory innervation) nor levels of group la innervation within muscle spindles were significantly altered in $b c /-2$ null compared to controls. Thus, the loss of sensory neurons seen within the DRG of bcl-2-null mice reflects $b c l-2$-dependent cell death in a class (or classes) of sensory neurons, perhaps group II neurons, which differentiate subsequently to group la neurons during embryonic development. $^{29}$

The development of muscle spindles is initiated upon the innervation of primary myotubes by proprioceptive (la) afferents. ${ }^{30}$ Arrival of sensory fibers is sufficient to trigger the differentiation of primary myofibers to early intrafusal $\left(\right.$ bag $_{2}$ ) fibers. ${ }^{31}$ It has been shown, at least for muscles of the hindlimb, that limiting concentrations of neurotrophin-3 (NT-3) produced within the developing myofibers regulate the outgrowth of these proprioceptive axons from the DRG to muscle spindles. ${ }^{32}$ As development proceeds, expression of NT-3 becomes restricted to intrafusal fibers within the developing spindle. Motor innervation of the muscle spindle occurs subsequent to the arrival of sensory fibers and the differentiation of the myotube. Consistent with this, survival of fusimotor neurons has been shown to be dependent upon the presence of la afferents and/or muscle spindles, as fusimotor neurons are not observed in mice lacking muscle spindles. ${ }^{27}$ By contrast, the near or total absence of motor innervation to muscle spindles does not prevent their differentiation or maintenance. ${ }^{27,33}$ This model is supported by our present work in $b c /-2^{-1-}$ mice, in which the loss of a substantial portion of fusimotor innervation did not significantly alter the number of muscle spindles within the hindlimb.

In support of the model in which $b c l-2$ differentially regulates the survival of gamma motor neurons, examinations of several lines of genetically modified mice that lack or overexpress factors known to act through Bcl-2 (bax and ntf3 null mutants, gdnf heterozygous mutants, MyoGDNF transgenic mice) exhibit significant differences in their gamma population. ${ }^{34-36}$ That gamma motor neurons require glial cell line-derived neurotrophic factor (GDNF) for survival derived from muscle spindles is suggested from studies in which significant losses of small diameter motor axons are observed in gdnf heterozygous animals. Similarly, transgenics ectopically expressing GDNF within skeletal muscle exhibit a substantial increase in small diameter motor axons within the $L 4$ ventral root, ${ }^{34,35}$ although the precise nature of the motor neurons contributing these axons is unclear. Analysis of the L4 ventral root in bax null mice reveals a significant increase in total numbers of myelinated axons, which is contributed exclusively by small diameter axons believed to be derived from gamma motor neurons. ${ }^{34,36}$ The greater relative increase in motor axons in bax null mice $(71 \%)$ compared to the reductions seen in bcl-2-null mice (24\%) likely reflects the greater potential for functional compensation by $\mathrm{Bcl}-2$ homologues (Bcl- $\mathrm{x}_{\mathrm{L}}, \mathrm{Mcl}-1, \mathrm{Bcl}-\mathrm{w}$, etc.) compared to Bax homologues (Bak, Bok, etc.) for motor neuron survival. Interestingly, it was also demonstrated that a substantial population of unmyelinated axons was rescued by bax deletion. ${ }^{34,37}$ While the ventral roots of adult wild-type mice normally contain very low numbers of unmyelinated axons, this is also true for our lineages of bcl-2-null mice (Supplementary Figure 1). Given the model proposed for $b c l-2$, and the nature of interactions between $\mathrm{Bcl}-2$ and $\mathrm{Bax}$, this finding is not surprising and paints a consistent picture of Bcl-2's action. Based upon the results in bcl-2-null mice, the mechanisms by which neurotrophic agents such as NT-3 and GDNF exert control over the development of specific motor populations, such as fusimotor neurons (and potentially alpha subgroups), can be clearly framed in a broader understanding of their influence by $b c /-2 / b a x$ interactions to regulate the process of $P C D$.

\section{Materials and Methods}

Animals. Bcl-2-null mice derived from two independent gene-targeting events ('bcl2DL', B13, and 'bcl2SK' Z3 - Jackson Labs) were obtained from the appropriate heterozygous intercrosses. The genotypes of all $b c l-2$ animals were confirmed by Southern blot analysis of genomic tail DNA or by PCR as described previously. ${ }^{19}$ Mice were raised under identical conditions in the same room of our animal facility, and killed for examination from P10 to P30. All experimental protocols conformed to Mount Sinai Hospital and University of Toronto animal colony care guidelines.

To assess the potential contribution of genetic background on any effects observed in bcl-2-null mice, two sublines of the initial 'bcl2DL' (mixed 129Sv: C57bl/ 6 background) stock were established through back-crossing onto either $129 \mathrm{~Sv} / \mathrm{Cp}$ (four generations) or C57BL/6J (five generations) to generate sublines 'bcl2DL-129' and 'bcl2DL-C57'. The results described obtained from comparative groups of wildtype, heterozygous, or $b c-$-2-null mice did not vary significantly from either of the sublines or comparative bcl2SK mice. The data shown in the paper represent data from bcl-2 homozygous or heterozygous littermates derived from C57BL/6J intercrosses (bcl2DL-C57 mice)

Retrograde tracing. P30 animals were anesthetized by intraperitoneal injection of a freshly prepared $2.5 \%$ solution of Avertin, at a dose of $0.2 \mathrm{ml}$ per $10 \mathrm{~g}$ body weight. Following the removal of overlying hair, a surgical incision was made in the skin and underlying fascia, and the desired nerve isolated. Following isolation of the nerve from surrounding tissue, a $5 \%$ solution of rhodamine dextran or Fast Dil (Molecular Probes) was pressure-injected into the indicated nerve $(250 \mathrm{nl})$ over a period of $3 \mathrm{~min}$. For sciatic nerves, the injection placement was $3 \mathrm{~mm}$ distal to the obdurator tendon. For facial nerves, the position of injection within the MFN is shown in Figure 5a. Following injection, incisions were closed with 6.0 
sutures and animals were allowed to recover for $48 \mathrm{~h}$, at which time they were killed and the appropriate tissues processed as indicated below.

Tissue preparation and analysis. Animals were deeply anesthetized with sodium pentobarbital (Somnitol, $80 \mathrm{mg} / \mathrm{kg}$ ) and perfused transcardially with $15 \mathrm{ml}$ of $100 \mathrm{mM}$ phosphate-buffered saline (PBS, pH 7.4), followed immediately by $50 \mathrm{ml}$ of freshly prepared $4 \%$ paraformaldehyde in PBS at $4{ }^{\circ} \mathrm{C}$. A 2-mm segment of the MFN was dissected and prepared as indicated below, as were muscles of the upper (flexor carpi ulnaris) and lower limbs (soleus and medial gastrocnemius). Whole brains were removed and the brainstem isolated from each animal. The spinal column corresponding to segments T12-L4 was exposed and isolated. Samples were subsequently post-fixed for a further $4 \mathrm{~h}$ in $4 \%$ paraformaldehyde in PBS at $4{ }^{\circ} \mathrm{C}$. At this point, the T12-L3 segment of the spinal cord, and the L4 dorsal and ventral roots were dissected out from each specimen. Spinal cords were processed for either cryostat or paraffin sections according to standard procedures. ${ }^{38}$ Spinal roots and muscle were processed as indicated below. Following processing, each sample block was given a coded identification number, so that data derived could be analyzed in a blinded manner.

For stereotactic analyses, brainstems containing the facial nucleus and the entirety of the T13-L2 segments of the lumbar spinal cord were processed as serial sets of $7 \mu \mathrm{m}$ paraffin sections. Following de-waxing, sections were stained with $0.1 \%$ thionin, dehydrated and mounted according to standard procedures. Facial motor counts were performed on every sixth section and total motor neuron numbers were assessed by the method of physical dissector (frame interval $=42 \mu \mathrm{m}){ }^{39}$ For counts of spinal cord ChAT-positive neurons and Nisslstained series (see below), the T13-L2 levels of spinal cords were isolated and cut as serial sets of $30 \mu \mathrm{m}$ cryostat sections. Total numbers of ChAT-positive motor neurons within the anterior horn were then evaluated at $300 \mu \mathrm{m}$ intervals $(n=4$ animals per genotype). In addition, these sections were stained with $1 \mu \mathrm{g} / \mathrm{ml}$ bisbenzamide (Hoechst 33258) to clarify the nuclei and nucleoli of motor neurons. Alternate sections in each series were stained with $0.1 \%$ thionin to compare Nissl counts of motor neuron number to those obtained with ChAT staining. For Nissl and ChAT series, no significant systematic variation in spinal motor neuron counts was observed between Nissl and ChAT, and hence only counts of ChAT-positive motor neurons are described herein. For all analyses, numeric counts were not corrected for split nucleoli. Statistical significance was assessed as indicated below (Statistical methods).

Thin sections. Nerve segments and muscle spindle samples were post-fixed in a solution of freshly prepared $2.5 \%$ glutaraldehyde in $100 \mathrm{mM}$ PBS (pH 7.4) for $4 \mathrm{~h}$ at $4{ }^{\circ} \mathrm{C}$; and rinsed free of glutaraldehyde and fixed in $1 \%$ osmium tetroxide buffered in PBS for $1 \mathrm{~h}$. Samples were dehydrated in a series of water/ethanol and ethanol/ propylene oxide baths. Following removal of propylene oxide, samples were embedded in spurr resin and baked at $50^{\circ} \mathrm{C}$ for $36 \mathrm{~h}$. A series of $1 \mu \mathrm{m}$ crosssections were then obtained and stained with $1 \%$ toluidine blue according to standard procedures. ${ }^{38}$ For spinal roots, thin sections were obtained at the point midway between the attached DRG and the spinal end of the nerve.

Histochemistry/immunohistochemistry. Following $3 \mathrm{~h}$ of post-fixation at $4{ }^{\circ} \mathrm{C}$, spinal cords and isolated muscles were placed in a solution of $30 \%$ sucrose and $0.1 \mathrm{M}$ PBS $(\mathrm{pH} 7.4)$ at $4{ }^{\circ} \mathrm{C}$ until equilibrated (12-15 h) and then frozen in 2-methyl butane at $-20^{\circ} \mathrm{C}$. Serial sets of $10-30 \mu \mathrm{m}$ cross-sections were obtained using a Reichardt-Jung Fridgocut cryostat at $-22^{\circ} \mathrm{C}$. Sections were mounted onto $2 \times$ gelatin-stubbed slides. Serial cryostat or microtome sections were stained with $0.1 \%$ thionin or processed for ChAT immunohistochemistry, using a goat anti-ChAT antibody (Chemicon AB144). For ChAT sections, peroxidase activity was first inhibited by incubating sections for $30 \mathrm{~min}$ in a solution of $100 \%$ methanol/3\% hydrogen peroxide. Nonspecific antibody binding was blocked by pre-incubation in $5 \%$ normal goat serum and $0.2 \%$ Tween-20 in PBS, pH 7.4, for $1 \mathrm{~h}$ at room temperature. Primary antibody was added at a dilution of $1: 800$ and sections were incubated overnight at $4^{\circ} \mathrm{C}$. Following washing, sections were incubated with biotinylated secondary antibody at a dilution of $1: 200$ for $2 \mathrm{~h}$ at room temperature followed by incubation with streptavidin-HRP at $1: 100$ for $1 \mathrm{~h}$ at room temperature (Vectastain ABC kit, PK-4005). Bcl-2 expression was detected by immunofluorescence using mouse anti-Bcl-2 antibody (DAKO M0887 1:20). Motor neurons were distinguished using anti-Islet-1/2 (1:1000) generously provided by the laboratory of Thomas Jessell. Fluorescent secondary antibodies $(1: 200)$ used were purchased from Molecular Probes.
Analysis of axon/nerve morphometry. The morphometry of nerve and muscle cross-sections were analyzed using a Leitz-Wetzlar microscope equipped with 25, 54, and 100 times objectives, a JVC model TK-1280U color video camera, and a $360^{\circ}$ rotating slide platform equipped with $X$ and $Y$ controllers. Axon and nerve areas were measured using a Leica Quantimet Q500MC system (Leica Canada, Willowdale, ON, Canada). The system was calibrated before and verified following each use using a Leica $10 \mu \mathrm{m}$ ruled calibration slide. Prior to analyzing each cross-section, a low-resolution $(25 \times)$ map was first generated and a hardcopy printed. This was used as a reference to place each of the individual nerve (analyzed at $100 \times$ ) or muscle (analyzed at $40 \times$ ) sectors in a given morphometric analysis. In each case, data were gathered for the nerve cross-section in its entirety, and sample data were coded and analyzed in a double-blinded manner.

Statistical methods. Statistical analyses were performed using Graphpad PRISM. Assessment of difference between means was determined by unpaired, two-tailed parametric Student's $t$-test with assumption of unequal variance. Null hypotheses were rejected at the 0.01 level. Results are expressed as mean \pm S.E.M. and were thus determined to be significant if $P<0.01$.

Acknowledgements. We thank Dr. Dennis Loh for the gift of $b c l-2$ null animals generated by his laboratory. We gratefully acknowledge the assistance of Maria Mendez for technical help and support on the morphometric analysis, Jackie Pittman, Douglas Holmyard, and Nancy Good for technical assistance on nerve thin sections. This work was supported by Rick Hansen Institute, ALS Society of Canada, and Canadian Institute of Health Research Institute. KH was supported by $\mathrm{CIHR/Rx \& D}$ Health Research Foundation.

1. Oppenheim RW. Cell death during development of the nervous system. Annu Rev Neurosci 1991; 14: 453-501.

2. Greenlund LJ, Korsmeyer SJ, Johnson Jr EM. Role of BCL-2 in the survival and function of developing and mature sympathetic neurons. Neuron 1995; 15: 649-661.

3. Akhtar RS, Ness JM, Roth KA. Bcl-2 family regulation of neuronal development and neurodegeneration. Biochim Biophys Acta 2004; 1644: 189-203.

4. Adams JM. Ways of dying: multiple pathways to apoptosis. Genes Dev 2003; 17 2481-2495.

5. Antonsson B. Bax and other pro-apoptotic Bcl-2 family 'killer-proteins' and their victim, the mitochondrion. Cell Tissue Res 2001; 306: 347-361.

6. Cory S, Adams JM. The BCL2 family: regulators of the cellular life-or-death switch. Nat Rev Cancer 2002; 2: 647-656.

7. Borner $\mathrm{C}$. The Bcl-2 protein family: sensors and checkpoints for life-or-death decisions. Mol Immunol 2003; 39: 615-647.

8. Leber B, Lin J, Andrews DW. Embedded together: the life and death consequences of interaction of the Bcl-2 family with membranes. Apoptosis 2007; 12: 897-911.

9. Tsujimoto Y, Shimizu S. Role of the mitochondrial membrane permeability transition in cell death. Apoptosis 2007; 12: 835-840.

10. Abe-Dohmae S, Harada N, Yamada K, Tanaka R. Bcl-2 gene is highly expressed during neurogenesis in the central nervous system. Biochem Biophys Res Commun 1993; 191 915-921.

11. Gonzalez-Garcia M, Garcia I, Ding L, O'Shea S, Boise LH, Thompson CB et al. bcl-x is expressed in embryonic and postnatal neural tissues and functions to prevent neuronal cell death. Proc Natl Acad Sci USA 1995; 92: 4304-4308.

12. Merry DE, Veis DJ, Hickey WF, Korsmeyer SJ. bcl-2 protein expression is widespread in the developing nervous system and retained in the adult PNS. Development 1994; 120 : 301-311.

13. Krajewska M, Mai JK, Zapata JM, Ashwell KW, Schendel SL, Reed JC et al. Dynamics of expression of apoptosis-regulatory proteins Bid, Bcl-2, Bcl-X, Bax and Bak during development of murine nervous system. Cell Death Differ 2002; 9: 145-157.

14. Yachnis AT, Giovanini MA, Eskin TA, Reier PJ, Anderson DK. Developmental patterns of BCL-2 and BCL-X polypeptide expression in the human spinal cord. Exp Neurol 1998; 150: 82-97.

15. Michaelidis TM, Sendtner M, Cooper JD, Airaksinen MS, Holtmann B, Meyer M et al. Inactivation of bcl-2 results in progressive degeneration of motoneurons, sympathetic and sensory neurons during early postnatal development. Neuron 1996; 17: 75-89.

16. Pinon LG, Middleton G, Davies AM. Bcl-2 is required for cranial sensory neuron survival at defined stages of embryonic development. Development 1997; 124: 4173-4178.

17. Martinou JC, Frankowski H, Missotten M, Martinou I, Potier L, Dubois-Dauphin M. Bcl-2 and neuronal selection during development of the nervous system. J Physiol, Paris 1994; 88: 209-211.

18. Veis DJ, Sorenson CM, Shutter JR, Korsmeyer SJ. Bcl-2-deficient mice demonstrate fulminant lymphoid apoptosis, polycystic kidneys, and hypopigmented hair. Cell 1993; 75 $229-240$. 
19. Nakayama K, Negishi I, Kuida K, Sawa H, Loh DY. Targeted disruption of Bcl-2 alpha beta in mice: occurrence of gray hair, polycystic kidney disease, and lymphocytopenia. Proc Nat Acad Sci USA 1994; 91: 3700-3704.

20. Cellerino A, Michaelidis T, Barski JJ, Bahr M, Thoenen $\mathrm{H}$, Meyer M. Retinal ganglion cell loss after the period of naturally occurring cell death in bcl-2-/- mice. Neuroreport 1999 10: $1091-1095$

21. Ohga Y, Zirrgiebel U, Hamner S, Michaelidis TM, Cooper J, Thoenen $\mathrm{H}$ et al. Cell density increases $\mathrm{Bcl}-2$ and $\mathrm{Bcl}-\mathrm{x}$ expression in addition to survival of cultured cerebellar granule neurons. Neuroscience 1996; 73: 913-917.

22. Dietz GP, Kilic E, Bahr M, Isenmann S. Bcl-2 is not required in retinal ganglion cells surviving optic nerve axotomy. Neuroreport 2001; 12: 3353-3356.

23. Bowden RE, Mahran ZY. The functional significance of the pattern of innervation of the muscle quadratus labii superioris of the rabbit, cat and rat. J Anat 1956; 90: 217-227.

24. Hashizume K, Kanda K, Burke RE. Medial gastrocnemius motor nucleus in the rat: agerelated changes in the number and size of motoneurons. J Comp Neurol 1988; 269: 425-430.

25. Dutton R, Yamada T, Turnley A, Bartlett PF, Murphy M. Regulation of spinal motoneuron differentiation by the combined action of Sonic hedgehog and neurotrophin 3. Clin Exp Pharmacol Phys 1999; 26: 746-748.

26. Kucera J, Walro JM, Reichler J. Role of nerve and muscle factors in the development of rat muscle spindles. Am J Anat 1989; 186: 144-160.

27. Ringstedt T, Copray S, Walro J, Kucera J. Development of fusimotor innervation correlates with group la afferents but is independent of neurotrophin-3. Brain Res 1998; 111 295-300.

28. Lance-Jones $\mathrm{C}$. Motoneuron cell death in the developing lumbar spinal cord of the mouse. Brain Res 1982; 256: 473-479.
29. Lawson SN, Biscoe TJ. Development of mouse dorsal root ganglia: an autoradiographic and quantitative study. J Neurocytol 1979; 8: 265-274.

30. Kucera J, Waldro JM. Treatment with beta bungarotoxin blocks muscle spindle formation in fetal rats. Development 1990; 110: 483-489.

31. Wang J, McWhorter DL, Walro JM. Stability of myosin heavy chain isoforms in selectively denervated adult rat muscle spindles. Anat Rec 1997; 249: 32-43.

32. Wright DE, Zhou L, Kucera J, Snider WD. Introduction of a neurotrophin-3 transgene into muscle selectively rescues proprioceptive neurons in mice lacking endogenous neurotrophin-3. Neuron 1997; 19: 503-517.

33. Kucera J, Walro JM. Formation of muscle spindles in the absence of motor innervation. Neurosci Lett 1992; 145: 47-50.

34. Buss RR, Gould TW, Ma J, Vinsant S, Prevette D, Winseck A et al. Neuromuscular development in the absence of programmed cell death: phenotypic alteration of motoneurons and muscle. J Neurosci 2006; 26: 13413-13427.

35. Whitehead J, Keller-Peck C, Kucera J, Tourtellotte WG. Glial cell-line derived neurotrophic factor-dependent fusimotor neuron survival during development. Mech Dev 2005; 122 : 27-41.

36. White FA, Keller-Peck CR, Knudson CM, Korsmeyer SJ, Snider WD. Widespread elimination of naturally occurring neuronal death in Bax-deficient mice. J Neurosci 1998; 18: $1428-1439$.

37. Sun W, Oppenheim RW. Response of motoneurons to neonatal sciatic nerve axotomy in Bax-knockout mice. Mol Cell Neurosci 2003; 24: 875-886.

38. Culling CFA. Handbook of Histopathology and Histochemistry Techniques 3rd edn. Butterworths: London, 1974.

39. Coggeshall RE, Lekan HA. Methods for determining numbers of cells and synapses: a case for more uniform standards of review. J Comp Neurol 1996; 364: 6-15.

Supplementary Information accompanies the paper on Cell Death and Differentiation website (http://www.nature.com/cdd) 